\title{
NRQCD matching coefficient at next-to-next-to-next-to-leading order
}

\section{Peter Marquard*}

TTP, Karlsruhe Institute for Technology (KIT)

E-mail: peter.marquard@kit.edu

We present a status report of the calculation of the matching coefficient of the vector current in non-relativistic QCD (NRQCD). The matching coefficient is one of the missing ingredients for a full next-to-next-to-next-to-leading order theory prediction for the total cross section for top anti-top production at thresold at a future linear collider.

36th International Conference on High Energy Physics

4-11 July 2012

Melbourne, Australia

* Speaker. 


\section{Introduction}

The precision of measurement of the top-quark mass at hadron colliders suffers from statistical limitations and a theoretically ill defined top-quark mass. It has been suggested in Ref. [1] that at a future linear collider the mass of the top quark can be measured with an accuracy of about $100 \mathrm{MeV}$ by studying the total cross section for $t \bar{t}$ production near threshold. To match the experimental uncertainty it is necessary to also have a reliable theory prediction for this quantity. The total cross section has been studied at next-to-next-to-leading order in Ref. [2]. The theory prediction is best done using an effective theory, non-relativistic QCD (NRQCD) [3, 4], which is suitable for the treatment of threshold and bound-state phenomena. Many building blocks for the complete third-order calculation have been calculated in recent years, ultra-soft effects have been considered in Refs. [5, 6], the three-loop static potential has been computed in Refs. [7, 8, 9] and in Ref. [10] a preliminary analysis of the top-quark threshold production cross section has been presented including also third-order potential effects.

One of the last missing pieces is the matching coefficient of the NRQCD vector current $c_{v}$, which connects the full theory, QCD, with the effective theory, NRQCD. The one- and two-loop results have been calculated in Refs. [11, 12, 13, 14]. At three-loops the fermionic contributions have been calculated in $[15,16]$. In the following we will review the results for the fermionic contributions and discuss the status of the calculation of the non-fermionic part.

\section{Calculation}

We start with the vector current in the full theory

$$
j^{\mu}=\bar{Q} \gamma^{\mu} Q
$$

where $Q$ denotes a heavy quark with mass $m_{Q}$. To obtain the corresponding current in NRQCD this has to be expanded in $1 / m_{Q}^{2}$, which gives at leading order

$$
\tilde{j}^{k}=\phi^{\dagger} \sigma^{k} \chi
$$

where $\phi$ and $\chi$ denote two-component Pauli spinors. The matching of the two theories is then obtained by requiring

$$
j^{k}=c_{v}(\mu) \tilde{j}^{k}+\mathscr{O}\left(\frac{1}{m_{Q}^{2}}\right),
$$

to all orders in perturbation theory, which defines the matching coefficient $c_{v}(\mu)$.

The matching coefficient is calculated best by considering renormalized onshell vertex corrections involving the currents $j^{\mu}$ and $\tilde{j}^{k}$

$$
Z_{2} \Gamma_{v}=c_{v} \tilde{Z}_{2} \tilde{Z}_{v}^{-1} \tilde{\Gamma}_{v}+\mathscr{O}\left(\frac{1}{m_{Q}^{2}}\right) .
$$

Here $Z_{2}$ and $\tilde{Z}_{2}$ denote the wave function renormalization constants of the quark in the full and effective theory, respectively. $Z_{2}$ has been calculated at three loops in Refs. [17, 18, 19], while 

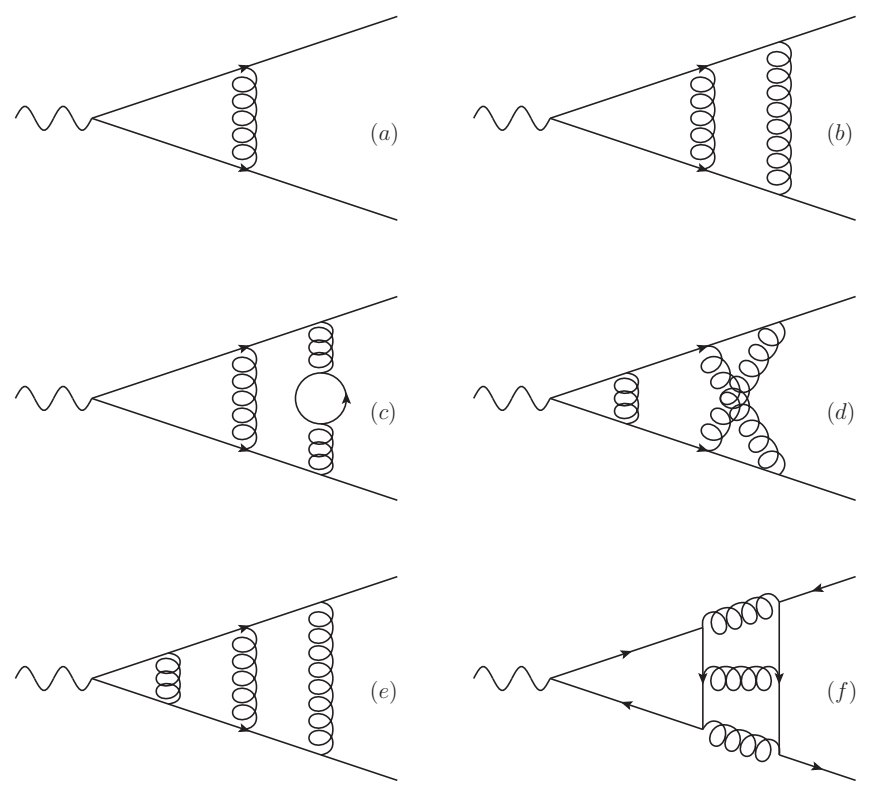

Figure 1: Sample diagrams contributing to $c_{v}$. Solid lines denote massive quarks and curly ones gluons.

$\tilde{Z}_{2}=1 . \tilde{Z}_{v}$ is the renormalization constant of the current $\tilde{j}^{k}$ and has been calculated in Ref. [20]. The vector current in the full theory does not require renormalization. All necessary renormalization constants are known and since both sides of Equation (2.4) receive the same soft, potential and ultra-soft contributions the calculation of vertex corrections can be performed at threshold. Typical diagrams appearing in the calculation are shown in Fig. 1.

The calculation is performed in a very automated way. The diagrams are calculated using qgraf [21] and mapped onto 80 topologies using q2e and exp [22, 23]. After applying suitable projectors and taking traces with FORM [24] the resulting scalar integrals are reduced to a small set of master integrals using the program CRUSHER [25], which implements Laporta's algorithm for solving integration-by-parts relations. After reduction the final step is the calculation of about 100 master integrals. For the calculation of the master integrals we employed the program FIESTA [26, 27], which is an implementation of the sector decomposition method for the calculation of Feynman integrals. Using FIESTA we calculated all required master integrals numerically ${ }^{1}$. To give an example for the accuracy that can be achieved using this approach we give the result for a typical master integral. The integrals given in Fig. 2 in graphical form can be evaluated to

$$
M_{1}=\frac{e^{3 \varepsilon \gamma_{E}}}{m_{Q}^{2}}(-22.72796(8)) \quad M_{2}=\frac{e^{3 \varepsilon \gamma_{E}}}{m_{Q}^{2}}(2.7327(3)+23.854(3) \varepsilon),
$$

where $\gamma_{E}=0.5772 \ldots$ is the Euler-Mascheroni constant. To ensure that all integrals are evaluated correctly and check the error estimate, we transformed the result by replacing all master integrals by a different set of integrals using integration-by-parts relations. The integrals in the new set were then again calculated using FIESTA. The results of both calculations agree within the error estimate.

\footnotetext{
${ }^{1}$ Master integrals like Fig. 1(f) resulting from singlet diagrams can at the moment not be calculated using F IESTA, but there contribution is expected to be small.
} 

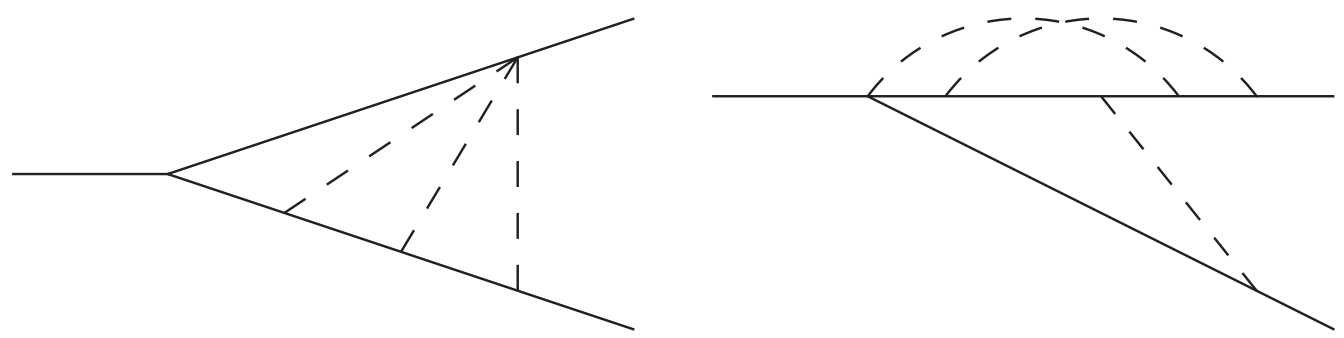

Figure 2: Non-trivial master integrals (left $M_{1}$, right $M_{2}$ ) needed for the calculation of $c_{v}$ at NNNLO. Solid and dashed lines denote massive and massless lines, respectively.

\section{Results}

Before turning to the matching coefficient itself a non trivial check can be performed. The renormalization constant $\tilde{Z}_{v}$ of the current in the effective theory has been calculated in the effective theory

$$
\begin{aligned}
& \tilde{Z}_{v}=1+\left(\frac{\alpha_{s}^{\left(n_{l}\right)}(\mu)}{\pi}\right)^{2} \frac{C_{F} \pi^{2}}{\varepsilon}\left(\frac{1}{12} C_{F}+\frac{1}{8} C_{A}\right)+\left(\frac{\alpha_{s}^{\left(n_{l}\right)}(\mu)}{\pi}\right)^{3} C_{F} \pi^{2} \\
& \times\left\{C_{F}^{2}\left[\frac{5}{144 \varepsilon^{2}}+\left(\frac{43}{144}-\frac{1}{2} \ln 2+\frac{5}{48} L_{\mu}\right) \frac{1}{\varepsilon}\right]+C_{F} C_{A}\left[\frac{1}{864 \varepsilon^{2}}+\left(\frac{113}{324}+\frac{1}{4} \ln 2+\frac{5}{32} L_{\mu}\right) \frac{1}{\varepsilon}\right]\right. \\
& +C_{A}^{2}\left[-\frac{1}{16 \varepsilon^{2}}+\left(\frac{2}{27}+\frac{1}{4} \ln 2+\frac{1}{24} L_{\mu}\right) \frac{1}{\varepsilon}\right]+T n_{l}\left[C_{F}\left(\frac{1}{54 \varepsilon^{2}}-\frac{25}{324 \varepsilon}\right)+C_{A}\left(\frac{1}{36 \varepsilon^{2}}-\frac{37}{432 \varepsilon}\right)\right] \\
& \left.+C_{F} \operatorname{Tn}_{h} \frac{1}{60 \varepsilon}\right\}+\mathscr{O}\left(\alpha_{s}^{4}\right),
\end{aligned}
$$

where $C_{A}=N_{c}, C_{F}=\left(N_{c}^{2}-1\right) /\left(2 N_{c}\right)$ and $T=1 / 2$ for a $\mathrm{SU}\left(N_{c}\right)$ gauge group and $L_{\mu}=\ln \left(\mu^{2} / m_{Q}^{2}\right)$, but can also be extracted from our calculation in the full theory. Comparing both results the accuracy of the calculation can be estimated. We find that the central values of the numerical calculation agree at the per-cent level with the analytical result for $\tilde{Z}_{v}$. This provides a first non-trivial check of the calculation.

We write the perturbative expansion of the matching coefficient in the form

$$
c_{v}=1+\frac{\alpha_{s}^{\left(n_{l}\right)}(\mu)}{\pi} c_{v}^{(1)}+\left(\frac{\alpha_{s}^{\left(n_{l}\right)}(\mu)}{\pi}\right)^{2} c_{v}^{(2)}+\left(\frac{\alpha_{s}^{\left(n_{l}\right)}(\mu)}{\pi}\right)^{3} c_{v}^{(3)}+\mathscr{O}\left(\alpha_{s}^{4}\right)
$$

and decompose $c_{v}^{(3)}$ according to the color structures as

$$
\begin{aligned}
c_{v}^{(3)}= & C_{F}\left[C_{F}^{2} c_{F F F}+C_{F} C_{A} c_{F F A}+C_{A}^{2} c_{F A A}+T n_{l}\left(C_{F} c_{F F L}+C_{A} c_{F A L}+T n_{h} c_{F H L}+T n_{l} c_{F L L}\right)\right. \\
& \left.+T n_{h}\left(C_{F} c_{F F H}+C_{A} c_{F A H}+T n_{h} c_{F H H}\right)\right]+ \text { singlet terms }
\end{aligned}
$$

At this point we will not present the result for the non-fermionic contribution, but only review the fermionic ones. The result for the non-fermionic contribution will be presented in Ref. [28]. The 
fermionic corrections have been calculated Refs. [15] and [16] and read

$$
\begin{aligned}
& c_{F F L}=46.7(1)+\left(-\frac{17}{12}+\frac{61}{36} \pi^{2}-\frac{2}{3} \pi^{2} \ln 2+\frac{1}{3} \zeta(3)\right) L_{\mu}+\frac{1}{18} \pi^{2} L_{\mu}^{2}, \\
& c_{F A L}=39.6(1)+\left(\frac{181}{54}-\frac{67}{432} \pi^{2}+\frac{5}{9} \pi^{2} \ln 2+\frac{13}{6} \zeta(3)\right) L_{\mu}+\left(\frac{11}{9}+\frac{1}{12} \pi^{2}\right) L_{\mu}^{2}, \\
& c_{F H L}=-\frac{557}{162}+\frac{26}{81} \pi^{2}+\left(-\frac{44}{27}+\frac{4}{27} \pi^{2}\right) L_{\mu}, \\
& c_{F L L}=-\frac{163}{162}-\frac{4}{27} \pi^{2}-\frac{11}{27} L_{\mu}-\frac{2}{9} L_{\mu}^{2}, \\
& c_{F F H}=-0.841(6)-\frac{1}{20} \pi^{2} L_{\mu}, \\
& c_{F A H}=-0.10(4)+\left(\frac{121}{27}-\frac{11}{27} \pi^{2}\right) L_{\mu}, \\
& c_{F H H}=-\frac{427}{162}+\frac{158}{2835} \pi^{2}+\frac{16}{9} \zeta_{3},
\end{aligned}
$$

where $L_{\mu}=\log \left(\mu^{2} / m_{Q}^{2}\right)$. Inserting the colour factors this evaluates numerically to

$$
c_{v}^{(3)} \approx-0.823 n_{l}^{2}+121.66(1) n_{l}-0.93(8)+\text { non-fermionic and singlet terms } .
$$

\section{Conclusion}

We presented a status report of the calculation of the non-fermionic corrections to the matching coefficient at next-to-next-to-leading order. The method of the calculation has been discussed and examples for the required master integrals have been presented. The validity of the method has been checked by reevaluating the renormalization constant $\tilde{Z}_{v}$. Furthermore, the result for the fermionic contributions have been reviewed.

\section{Acknowledgements}

This work has been supported by the DFG through the SFB/TR 9 "Computational Particle Physics". We like to thank J. Piclum, D. Seidel and M. Steinhauser for collaboration and M. Steinhauser for reading of the manuscript.

\section{References}

[1] M. Martinez and R. Miquel, Eur. Phys. J. C 27 (2003) 49 [arXiv:hep-ph/0207315].

[2] A. H. Hoang et al., Eur. Phys. J. directC 2 (2000) 1 [arXiv:hep-ph/0001286].

[3] W. E. Caswell and G. P. Lepage, Phys. Lett. B 167 (1986) 437.

[4] G. T. Bodwin, E. Braaten and G. P. Lepage, Phys. Rev. D 51 (1995) 1125 [Erratum-ibid. D 55 (1997) 5853] [arXiv:hep-ph/9407339].

[5] M. Beneke, Y. Kiyo and A. A. Penin, Phys. Lett. B 653 (2007) 53 [arXiv:0706.2733 [hep-ph]].

[6] M. Beneke and Y. Kiyo, Phys. Lett. B 668 (2008) 143 [arXiv:0804.4004 [hep-ph]]. 
[7] A. V. Smirnov, V. A. Smirnov and M. Steinhauser, Phys. Lett. B 668 (2008) 293 [arXiv:0809.1927 [hep-ph]].

[8] A. V. Smirnov, V. A. Smirnov and M. Steinhauser, Phys. Rev. Lett. 104 (2010) 112002 [arXiv:0911.4742 [hep-ph]].

[9] C. Anzai, Y. Kiyo and Y. Sumino, Phys. Rev. Lett. 104 (2010) 112003 [arXiv:0911.4335 [hep-ph]].

[10] M. Beneke, Y. Kiyo, A. Penin and K. Schuller, In the Proceedings of 2007 International Linear Collider Workshop (LCWSO7 and ILCO7), Hamburg, Germany, 30 May - 3 Jun 2007, pp TOP01 [arXiv:0710.4236 [hep-ph]].

[11] G. Källen and A. Sarby, K. Dan. Vidensk. Selsk. Mat.-Fis. Medd. 29, N17 (1955) 1.

[12] A. Czarnecki and K. Melnikov, Phys. Rev. Lett. 80 (1998) 2531 [arXiv:hep-ph/9712222].

[13] M. Beneke, A. Signer and V. A. Smirnov, Phys. Rev. Lett. 80 (1998) 2535 [arXiv:hep-ph/9712302].

[14] B. A. Kniehl, A. Onishchenko, J. H. Piclum and M. Steinhauser, Phys. Lett. B 638 (2006) 209 [arXiv:hep-ph/0604072].

[15] P. Marquard, J. H. Piclum, D. Seidel and M. Steinhauser, Nucl. Phys. B 758 (2006) 144 [arXiv:hep-ph/0607168].

[16] P. Marquard, J. H. Piclum, D. Seidel and M. Steinhauser, Phys. Lett. B 678 (2009) 269 [arXiv:0904.0920 [hep-ph]].

[17] D. J. Broadhurst, N. Gray and K. Schilcher, Z. Phys. C 52 (1991) 111.

[18] K. Melnikov and T. van Ritbergen, Nucl. Phys. B 591 (2000) 515 [arXiv:hep-ph/0005131].

[19] P. Marquard, L. Mihaila, J. H. Piclum and M. Steinhauser, Nucl. Phys. B 773 (2007) 1 [arXiv:hep-ph/0702185].

[20] B. A. Kniehl, A. A. Penin, M. Steinhauser and V. A. Smirnov, Phys. Rev. Lett. 90 (2003) 212001 [Erratum-ibid. 91 (2003) 139903(E)] [arXiv:hep-ph/0210161].

[21] P. Nogueira, J. Comput. Phys. 105, 279 (1993).

[22] R. Harlander, T. Seidensticker and M. Steinhauser, Phys. Lett. B 426 (1998) 125 [hep-ph/9712228].

[23] T. Seidensticker, hep-ph/9905298.

[24] J. A. M. Vermaseren, math-ph/0010025.

[25] D. Seidel and P. Marquard, unpublished.

[26] A. V. Smirnov and M. N. Tentyukov, Comput. Phys. Commun. 180 (2009) 735 arXiv:0807.4129 [hep-ph].

[27] A. V. Smirnov, V. A. Smirnov and M. Tentyukov, Comput. Phys. Commun. 182 (2011) 790 [arXiv:0912.0158 [hep-ph]].

[28] P. Marquard, J.K. Piclum, D. Seidel and M. Steinhauser, in preparation. 